\title{
Helminths and microbes within the vertebrate gut - not all studies are created equal
}

\begin{tabular}{|r|l|}
\hline Journal: & Parasitology \\
\hline Manuscript ID & PAR-2019-0112.R2 \\
\hline Danuscript Type: & Review \\
\hline Author: & 16-Jun-2019 \\
\hline & $\begin{array}{l}\text { Complete List of Authors: } \\
\text { Peachey, Laura; Cambridge Veterinary School, Department of } \\
\text { Veterinary Medicine } \\
\text { Jenkins, Timothy; Cambridge Veterinary School, Department of } \\
\text { Veterinary Medicine } \\
\text { Scotti, Riccardo; Cambridge Veterinary School, Department of Veterinary } \\
\text { Medicine } \\
\text { Cantacessi, Cinzia; Cambridge Veterinary School, Department of } \\
\text { Veterinary Medicine; University of Cambridge }\end{array}$ \\
\hline Key Words: & $\begin{array}{l}\text { Helminth-microbiota interactions, Microbial richness, Microbial diversity, } \\
\text { Human studies, Gastrointestinal parasites }\end{array}$ \\
\hline & \\
\hline
\end{tabular}


Invited review

\section{Helminths and microbes within the vertebrate gut - not all studies are created equal

\begin{abstract}
Alba Cortés ${ }^{1}$, Laura E. Peachey ${ }^{1,2}$, Timothy P. Jenkins ${ }^{1}$, Riccardo Scotti ${ }^{1}$ and Cinzia Cantacessi ${ }^{1}$
${ }^{1}$ Department of Veterinary Medicine, University of Cambridge, Madingley Road CB3 OES, Cambridge, United Kingdom
\end{abstract}
${ }^{2}$ Bristol Veterinary School, Faculty of Health Sciences, University of Bristol, Langford House, Langford, BS40 5DU, Bristol, United Kingdom

Running title: Helminth-microbiota interactions in the human gut

\section{Corresponding author:}

\section{Cinzia Cantacessi}

Department of Veterinary Medicine, University of Cambridge, Madingley Road CB3 OES, Cambridge, United Kingdom

Tel. +44 (0) 1223760541

Fax. +44 (0)1223 337610

E-mail: cc779@cam.ac.uk

4,648 words 


\section{SUMMARY}

30 The multifaceted interactions occurring between gastrointestinal (GI) parasitic helminths and the 31 host gut microbiota are emerging as a key area of study within the broader research domain of 32 host-pathogen relationships. Over the past few years, a wealth of investigations has demonstrated 33 that GI helminths interact with the host gut flora, and that such interactions result in modifications 34 of the host immune and metabolic statuses. Nevertheless, whilst selected changes in gut microbial 35 composition are consistently observed in response to GI helminth infections across several host36 parasite systems, research in this area to date is largely characterised by inconsistent findings.

37 These discrepancies are particularly evident when data from studies of GI helminth-microbiota 38 interactions conducted in humans from parasite-endemic regions are compared. In this review, we provide an overview of the main sources of variance that affect investigations on human-

40 helminth-gut microbiota interactions and propose a series of methodological approaches that, 41 whilst accounting for the inevitable constraints of human fieldwork, are aimed at minimising 42 confounding factors and draw biologically meaningful interpretations from highly variable 43 datasets. 


\section{INTRODUCTION}

A plethora of experimental evidence supports a key role of infections by gastrointestinal (GI) helminth parasites in shaping the composition of the vertebrate gut microbiota, with significant implications for local and systemic host immunity (reviewed by Brosschot and Reynolds, 2018). For instance, recent studies have partly attributed the parasite-associated qualitative and/or quantitative alterations to host GI microbial profiles to the ability of GI helminths to stimulate the initial onset of T-regulatory (Treg) immune responses (cf. Cantacessi et al. 2014; Reynolds et al. 2014; Giacomin et al. 2015, 2016; Zaiss et al. 2016). On the other hand, other studies have reported associations between acute helminth infections and gut microbiota imbalances (= dysbiosis) characterised by significant expansion of populations of putative pro-inflammatory bacteria (e.g. Rausch et al. 2013; Jenkins et al. 2018a; Schneeberger et al. 2018a); these observations have lent credit to the hypothesis that helminth-associated alterations of gut microbiota composition may lead to both localised and systemic consequences for the host organism, that include immunopathology and exacerbated malnutrition in at-risk subjects from parasite-endemic areas (reviewed by Glendinning et al. 2014; Houlden et al. 2015; Cattadori et al. 2016).

Over the past decade, newly acquired knowledge of the impact that GI helminth infections exert on the vertebrate gut microbial composition and metabolism has contributed to a better understanding of parasite systems biology and host-pathogen interactions (reviewed by Peachey et al. 2017; Leung et al. 2018; Rapin and Harris et al. 2018), and has been proposed as a first step towards the identification and development of novel strategies of parasite control based on the targeted manipulation of the host gut microbiota (cf. Peachey et al. 2017). Nevertheless, for humans in particular, progress in this field of research is greatly impaired by the impact of several confounding factors that inevitably affect studies conducted in naturally infected individuals (Mutapi, 2015; Chabé et al. 2017). In this review, we summarise current knowledge of GI helminth-microbiome interactions in humans under natural conditions of infection, identify similarities and differences between datasets and provide an overview of the confounding factors that may affect the interpretation of findings. 

SCENARIOS

In endemic areas for helminthiases, the vast majority of infected individuals harbour multiple helminth species, often occupying different niches of the host organism (Hotez et al. 2010). Whilst polyparasitism is often regarded as a major confounding factor in investigations of parasite-microbiota interactions conducted in humans under natural conditions of infection (Cooper et al. 2013; Jenkins et al. 2017; Martin et al. 2018; Rosa et al. 2018), findings from these studies are key to assessing the impact that GI helminths exert on gut microbiota homeostasis in a 'real-world' scenario. Nevertheless, several factors should be considered when interpreting results obtained from individuals infected by multiple helminth species. First, anthropometric (e.g. age and gender) and anthropologic variables (e.g. ethnicity, diet and occupation) are well known to profoundly impact the 'baseline' composition of the human gut microbiota (Sekirov et al. 2010; Yatsunenko et al. 2012) (cf. Fig. 1); therefore, the enrolment of large cohorts of individuals is often necessary in order to achieve sufficient statistical power and avoid uninformative and/or misleading results (Kelly et al. 2015). However, in many studies, the number of individuals enrolled and samples analysed is inevitably dictated by logistical and financial constraints. In these instances, population-related variables that impact gut microbiota composition may contribute substantially to inconsistencies among findings from different studies (cf. Fig. 1). For instance, a negative association between colonisation by the whipworm Trichuris trichiura and the abundance of bacteria belonging to the genus Prevotella in the faeces of infected individuals has been reported in two separate studies conducted in Malaysia (Lee et al. 2014; Ranaman et al. 2016), while other studies conducted in Ecuador, and Liberia and Indonesia, respectively, have failed to identify significant variations in faecal populations of Prevotella in individuals either solely infected by T. trichiura or co-infected with other species of soiltransmitted helminths (STHs) (Cooper et al. 2013; Martin et al. 2015; Rosa et al. 2018). microbial profiles of helminth-harbouring individuals that were specifically associated to single 
infections with the hookworm Necator americanus, the roundworm Ascaris lumbricoides or $T$.

trichiura, such features were inconsistent between two independent cohorts of helminth-infected volunteers from Liberia and Indonesia; this discrepancy suggests that other yet undetermined environmental factors may contribute to qualitative and quantitative alterations of the gut microbial profiles of helminth-infected individuals from different geographical areas. In contrast, an association between the abundance of selected bacterial taxa and infections by one or more STHs could be consistently detected in samples from both Liberian and Indonesian cohorts (Rosa et al. 2018). These taxa included bacteria belonging to the genera Olsenella and Allobaculum, which were expanded in the gut microbiota of helminth-infected individuals when compared to that of uninfected controls. To the best of our knowledge, the study by Rosa et al. (2018) was the first to report a link between infections by STHs and the abundance of these bacterial genera in the human gut. Interestingly, in mice suffering from metabolic syndrome, administration of probiotics was followed by expansion of populations of Olsenella and/or Allobaculum, and a reduction in systemic and/or local gut inflammatory responses (Wang et al. 2015). Moreover, Allobaculum spp. are putative producers of anti-inflammatory short-chain fatty acids (Greetham et al. 2004), and are severely reduced in the gut of mice genetically predisposed to spontaneous colitis (Pérez-Muñoz et al. 2014). This knowledge led Rosa et al. (2018) to hypothesize that these bacteria may play a yet undetermined role in the anti-inflammatory properties of parasitic helminths, and reinforce the proposition that the interactions between hosts, parasites and gut microbiota are multidirectional and should be approached in a holistic manner (e.g. Cortés et al. 2018; Leung et al. 2018). Interestingly, in contrast to evidence acquired in human hosts, a negative association between the genus Allobaculum and colonisation by GI helminths has been observed in a mouse model of chronic trichuriasis (Holm et al. 2015), in which Th1-mediated immune responses are dominant (reviewed by Cliffe and Grencis, 2004), as well as in mice with patent infection by the blood fluke Schistosoma mansoni (Jenkins et al. 2018a), in which migrating eggs are responsible for the onset of marked Th2-mediated inflammatory responses (reviewed by Pearce and MacDonald, 2002). The immune-molecular mechanisms via which members of the genus Allobaculum may regulate local and systemic inflammation are still unclear (Greetham et 

al. 2004; Pérez-Muñoz et al. 2014; Wang et al. 2015). Nonetheless, current data showing reductions in populations of Allobaculum alongside helminth-associated gut inflammation supports the hypothesis raised by Rosa et al. (2018); in the future, rodent models of GI helminth infections whose gut microbiota is deprived of, and subsequently recolonised with, the genus Allobaculum could be exploited to investigate the potential involvement of these bacteria in parasite-mediated immunomodulation.

Beside the intrinsic variability of the human gut microbiota, studies conducted under natural conditions of helminth colonisation are likely to be affected by factors linked to the different combinations of infecting species and their relative abundances. For instance, in a study conducted in a cohort of Ecuadorian children, the specific features detected in the gut microbial profiles of subjects co-infected with $T$. trichiura and A. lumbricoides could not be identified in the microbiota of Trichuris-only infected individuals (Cooper et al. 2013). Similarly, selected microbial features that were observed in studies conducted in human volunteers with monospecific infections with, for instance, A. lumbricoides, could not be detected in the gut microbiota of subjects harbouring the same parasite alongside other helminth species (e.g. T. trichiura and N. americanus) (Rosa et al. 2018), thus suggesting that a complex interplay exists between the host gut and its macro- and microbiota, that might be difficult to replicate in experimental settings. Furthermore, current evidence obtained from animal models of helminth infections indicates that worm burdens can impact the nature and/or the magnitude of parasite-associated alterations in gut microbial composition (Wu et al. 2012; Peachey et al. 2018). Nevertheless, such evidence is not yet available for human infections, in which parasite burdens may range from low to very high in endemic areas (Barbour and Kafetzaki, 1991; Churcher et al. 2005).

Another frequent constraint of investigations conducted in cohorts of human subjects with natural helminth infections is the limited availability of 'genuine' negative controls, i.e. individuals from the same communities of parasite-infected subjects who lack previous exposure to infections by parasitic helminths. Instead, individuals with no evidence of patent helminth infections are inevitably enrolled as control subjects (e.g. Cooper et al. 2013; Lee et al. 2014; Jenkins et al. 
2017; Rosa et al. 2018); nevertheless, studies in helminth-infected individuals subjected to anthelmintic treatment, as well as in primates and pigs exposed to Trichuris spp., have shown that parasite-associated alterations in gut microbial communities can persist, at least partly, in absence of active infections (Broadhurst et al. 2012; Wu et al. 2012; Cooper et al. 2013; Kay et al. 2015; Schneeberger et al. 2018a). These data call for caution when interpreting differences between the gut microbial profiles of helminth-infected and uninfected volunteers from the same communities. In addition, patent infections are often diagnosed using stool-based microscopic methods, that are known for their relatively low sensitivity and that may yield false negative results, e.g. in case of intermittent shedding of eggs and/or larvae (O’Connell and Nutman, 2016). Recently, Rosa et al. (2018) used quantitative real-time PCR to diagnose STH infections in individuals subjected to gut microbiota profiling, indicating that this technique may represent a robust and sensitive alternative to microscopic methods, since it provides users with the ability to semi-quantify burdens of different helminth species from minute amounts of DNA template. However, in spite of their higher sensitivity, molecular methods rely on the use of primers that selectively target the parasite species of interest, thus impairing the simultaneous detection of potential (asymptomatic or subclinical) co-infections with other helminth and/or non-helminth pathogens (O'Connell and Nutman, 2016). Indeed, the impact of protozoa on the gut microbial diversity and composition has been clearly demonstrated in humans and other vertebrates (reviewed by Chabé et al. 2017; Stensvold and van der Giezen, 2018). Furthermore, a recent study conducted in a cohort of Colombian schoolchildren reported common features in the faecal microbial composition of subjects co-infected with helminths and protozoans and mono-parasitized with the flagellate Giardia intestinalis compared to uninfected individuals (Toro-Londono et al. 2019). Whilst the mechanisms via which each group of parasites alters the host gut flora, as well as the nature of such alterations, are yet to be determined, these findings support the need to conduct additional diagnostic tests on stool samples from helminth-infected cohorts, as well as the corresponding uninfected subjects, in order to rule out the influence of concomitant bacterial, viral and/or protozoan infections that may be responsible for the changing gut microbial profiles of these individuals (cf. Chabé et al. 2017). 
Nevertheless, in spite of the several confounding factors outlined above (cf. Fig. 1), observational studies in helminth endemic areas have proven useful for the identification of significant associations between parasite colonisation and the gut microbial profiles of humans under natural conditions of infection. Importantly, studies conducted in these communities provide excellent opportunities to evaluate the effect(s) that parasite removal (e.g. via the administration of broadspectrum anthelmintics) exert(s) on the gut microbiota of previously infected individuals, thus contributing cues to understand the causality of helminth-microbiota relationships.

\section{IMPACT OF DEWORMING ON THE HUMAN GUT MICROBIOTA}

The implementation of mass drug administration programmes in endemic areas for STHs and schistosomiasis offers opportunities to elucidate potential mechanisms via which parasitic helminths modulate the host gut microbiota. For instance, qualitative and quantitative changes in gut microbial profiles that are caused by direct interactions between parasites and gut bacteria may be expected to rapidly reverse following parasite removal, whilst long-lasting alterations are likely to result from indirect interplay mediated by the host immune system (Houlden et al. 2015; Su et al. 2018). Nevertheless, such investigations are also generally constrained by the presence of several confounding factors that include not only the host- and parasite-dependent variables outlined above, but also variations linked to the use of different drugs and treatment regimes (Schneeberger et al. 2018b), as well as time of sampling post-anthelmintic treatment (Houlden et al. 2015) (Fig. 1). The latter in particular may profoundly affect findings from these studies, as the presence of tissue lesions caused by e.g. parasite feeding activity and location (e.g. bloodfeeders $v s$. non blood-feeders and luminal $v s$. tissue dwellers) are likely to influence the timespan between helminth removal and microbiome recovery (reviewed by Leung et al. 2018). Moreover, for ethical reasons, data from these experiments is often biased by the lack of placebo-treated control groups. These limitations may be at least partially responsible for the differences between findings from studies aimed at elucidating the effect of deworming on the gut microbiota of helminth-infected volunteers; notwithstanding, it is worth noting that, in instances where 

deworming-associated changes in human gut microbial profiles were detected, these were generally moderate (Ramanan et al. 2016; Martin et al. 2018; Schneeberger et al. 2018b).

Consistent with this, a recent study conducted on faecal samples collected from a rural community in Indonesia reported that the composition of the gut microbiota of individuals repeatedly treated with either albendazole or placebo (for 21 months) resembled that of samples collected from the same subjects prior to treatment, rather than that of uninfected controls (Rosa et al. 2018). Moreover, a parallel investigation conducted on the same cohort of individuals detected reduced populations of Prevotella in albendazole-treated subjects in which complete deworming did not occur, compared to placebo-treated individuals with patent helminth infections (Martin et al. 2018). Intriguingly, failure of albendazole treatment was accompanied by a dominance of $T$. trichiura (over other helminth species) in these subjects, while placebo-treated individuals maintained a diverse macrobiota (i.e. multiple helminth infections); hence, differences in the composition of the GI macrobiota (i.e. species present and their relative abundances) between albendazole- and placebo-treated individuals could account for variations in the composition of the intestinal microflora of these subjects (Martin et al. 2018). Significant associations between colonisation by T. trichiura and Prevotella abundance were not observed in the Indonesian cohort (Martin et al. 2018; Rosa et al. 2018). However, negative associations between whipworm infections and Prevotella abundance had been detected previously in two independent studies conducted in Malaysia (Lee et al. 2014; Ramanan et al. 2016). In particular, Ramanan and coauthors (2016) observed that, following albendazole treatment, expansion of Prevotella populations in the human faecal microbiota was related to reduced T. trichiura faecal egg counts. In contrast, no significant associations between helminth infection and abundance of bacteria belonging to the genus Prevotella was reported in a study investigating the impact of parasite colonisation and successful treatment with a combination of albendazole and ivermectin on the faecal microbial profiles of a cohort of Trichuris-infected children from Ecuador (Cooper et al. 2013), nor in a group of helminth-infected adults from Sri Lanka treated with pyrantel pamoate (Jenkins et al. 2017). Similarly, no qualitative or quantitative changes to faecal microbial 
composition were observed in two cohorts of schoolchildren from Côte d'Ivoire and Zimbabwe

237

infected by $S$. mansoni and $S$. haematobium, respectively, following treatment with praziquantel (Kay et al. 2014; Schneeberger et al. 2018a). However, successful elimination of S. mansoni was associated with a higher abundance of Fusobacterium spp. pre-treatment, as well as 24 hrs posttreatment (Schneeberger et al. 2018a).

Whilst drug administration in endemic regions may result in effective elimination of helminth infections, potential co-infecting protozoan parasites are not susceptible to anthelmintic treatment; this, together with the sub-standard hygienic and sanitary conditions that generally characterise these areas and that result in continuous re-exposure to infective helminth developmental stages (Campbell et al. 2018), impairs the full assessment of the consequences of helminth removal on the composition of the human gut microbiota. To the best of our knowledge, thus far, a single study has investigated the effects of chronic infections by a GI helminth, Strongyloides stercoralis, and anthelmintic treatment on the composition of the faecal microbiota and metabolome of humans from a non-endemic area of Europe, where parasite transmission had been interrupted (Jenkins et al. 2018b). Treatment with ivermectin resulted in compositional changes of the faecal microbiota (analysed 6 months post-treatment), which partially resembled that of uninfected control subjects (Jenkins et al. 2018b); in particular, alpha diversity [= a measure of the number of bacterial species present in a given microbial community (richness) and their relative abundance (evenness)] was reduced in the microbiota of individuals post-treatment (although statistical significance was not achieved) and accompanied by expanded populations of potentially pathogenic bacteria (Jenkins et al. 2018b). In addition, the faecal metabolic profiles obtained from samples collected post-ivermectin treatment shared features with both those obtained from samples collected pre-treatment and from uninfected controls (Jenkins et al. 2018b); this observation led Jenkins et al. (2018b) to hypothesise that, following parasite removal and over time, both gut microbiota and metabolome may revert to the original pre-infection state. Multiple factors, including but not limited to those outlined above, may contribute to the discrepancies observed between the findings from this work and those that reported no or minor 

al. 2013; Ramanan et al. 2016; Martin et al. 2018; Rosa et al. 2018; Schneeberger et al. 2018a,b).

Despite the limitations outlined above, studies of GI helminth-microbiota relationships conducted in endemic areas for helminthiases have provided repeated evidence of the perturbations that parasites and anthelmintic treatment exert on the equilibrium of resident populations of gut bacteria and on gut homeostasis. However, the identification of common signatures across studies remains key to designing future experiments, e.g. in animal models of helminth infections, that may assist the elucidation of the mechanisms that underpin the interactions between GI helminths, the gut microbiota and the host immune system.

\section{DO COMMON SIGNATURES EXIST ACROSS STUDIES OF HOST-HELMINTH-} MICROBIOTA INTERACTIONS?

The identification of gut microbial signatures that occur reproducibly across several host-GI helminth systems is crucial for designing novel anti-helminth intervention strategies based on the manipulation of the gut microbiota (Peachey et al. 2017). Studies conducted in animal models of helminth infections are expected to assist the identification of such signatures, as well as the direct (i.e. parasite-mediated) and/or indirect (i.e. immune-mediated) mechanisms that govern helminthmicrobiota interactions (Cortés et al. 2018); nevertheless, the inconsistencies that characterise studies of helminth-microbiota relationships published to date make such a task highly challenging. Indeed, for patterns to be identified, fluctuations in selected populations of gut microbes must be interpreted in light of the physical and immunological alterations of the mucosal environment in which such alterations occur (Leung et al. 2018). For instance, expanded populations of Lactobacillaceae have been repeatedly detected following infection with several species of parasitic helminths in several host species (Reynolds et al. 2014; Duarte et al. 2015; Holm et al. 2015; Houlden et al. 2015; Cattadori et al. 2016; Jenkins et al. 2018a; Kim et al. 2018), and could thus be considered as a 'consistent alteration' in gut microbiota composition upon helminth colonisation. However, key differences exist between host-parasite pairs investigated in the studies that have reported such an outcome. Indeed, whilst populations of 
Lactobacillaceae promote regulatory responses in mice infected by Heligmosomoides polygyrus bakeri (Reynolds et al. 2014), a lack of correlation between Lactobacillaceae abundance and Treg populations has been observed in other host-parasites systems, such as mice chronically infected with T. muris and rabbits infected with Trichostrongylus retortaeformis, in which the expansion of populations of gut Lactobacillaceae upon helminth infection occurs in an environment dominated by Th1-mediated immune responses (Holm et al. 2015; Houlden et al. 2015; Cattadori et al. 2016). These differences suggest that alternative mechanisms may regulate the differentiation and development of adaptive immune responses in each host-parasite system (Houlden et al. 2015), and thus that similar alterations in gut microbiota composition may result in different consequences that are dependent on the microenvironment where these changes occur. Notwithstanding, the interactions between hosts, helminths and the gut microbiota are likely multifaceted and multidirectional, and therefore the potential consequences that selected compositional changes in gut microbiota exert on host homeostasis are only one aspect of these complex interplay. For instance, a common yet undetermined mechanism may determine the expansion of Lactobacillaceae in the gut of helminth-infected hosts.

On the other hand, apparent 'contradictory' findings across studies may result from fundamental differences between gut compartments under investigation. For instance, Prevotella spp. was expanded in the abomasum and faeces of sheep infected by abomasal trichostrongyles (i.e. Haemonchus contortus and Teladorsagia circumcincta; Li et al. 2016; Cortés et al. in preparation), whilst the same taxa were reduced in the faeces of a range of host species, including mice, humans and horses, infected by nematodes residing in the large intestine, i.e. Trichuris spp. and cyathostomins, respectively (Lee et al. 2014; Houlden et al. 2015; Peachey et al. submitted). It must be noted, however, that whilst increased abomasal pH favours Prevotella overgrowth in the abomasum (De Nardi et al. 2016; Li et al. 2016), the same taxa are likely to be exposed to a dramatically different microenvironment in the large intestine that may determine the contraction of these bacterial groups. In addition, given the functional dissimilarities between the abomasal and colonic microbiota, such alterations are expected to result in fundamentally different 
317 outcomes for the homeostasis of each of these gut compartments (Ley et al. 2008), and hence 318 comparisons are, in our opinion, unwarranted.

319 In parallel to species of bacteria with functions that may vary depending on the gut compartment, multiple taxa share the same functions in different microenvironments (Lozupone et al. 2012); therefore, it is plausible that, even though inconsistencies are detected across studies, these may result in similar functional alterations in the host-parasite pairs being compared. For instance, recent studies in mouse and humans infected with $S$. mansoni have reported the expansion of different genera of bacteria with pro-inflammatory functions in the gut microbiota of the respective hosts (Jenkins et al. 2018a; Schneeberger et al. 2018a). These observations lend credit to the hypothesis that the functional role of the gut microbiota in helminth infections could be far less 'diverse' than the taxonomic associations reported thus far. For this hypothesis to be confirmed or confuted, a better understanding of the function(s) of each bacterial taxon inhabiting the different gut compartments in a range of host species is needed. To this aim, the integration of metagenomic, metabolomic and metatranscriptomic technologies, alongside traditional microbiology and microscopy techniques, may assist to achieve a holistic picture of the impact of GI helminth infections on the functions of the human gut microbiota, and its significance for disease pathophysiology and overall host health (Wang et al. 2015).

\section{CURRENT NEEDS AND FUTURE DIRECTIONS}

Understanding the complex interactions between GI helminths and their vertebrate hosts is pivotal for advancing our knowledge of the fundamental biology of these parasites and the diseases they cause (see Peachey et al. 2017; Leung et al. 2018; Rapin and Harris et al. 2018 for reviews). Whilst the role of the gut microbiota in host-parasite relationships has long been overlooked, current knowledge of the key roles that resident bacteria play in host health and disease, together with recent technical advancements for microbiota profiling, have boosted research is this area.

341 This is currently leading to increasing evidence of a role for the gut microbiota in the immune

342 regulatory properties of helminth parasites (Cantacessi et al. 2014; Reynolds et al. 2014; 343 Giacomin et al. 2015, 2016; Zaiss et al. 2016). In addition, data collected to date points towards 
a likely role of the gut microflora in the immunopathology of selected GI helminth infections that awaits experimental validation. Trying to untangle the relevance of particular fluctuations of specific bacterial taxa on infection outcome is challenging; nevertheless, currently available data suggest that low-intensity, long-term helminth infections are commonly linked to high microbial diversity and predominance of bacteria typically associated with gut health. Conversely, highintensity, acute infections are often associated to gut dysbiosis, characterised by reduced alpha diversity and an increase in pro-inflammatory and often opportunistic pathogens (Peachey et al. 2017). However, for this knowledge to be exploited in translational studies, further investigations in both natural and experimental settings are needed to distinguish spurious results from genuine helminth-microbiota associations (Peachey et al. 2017), and mechanistic studies in animal models of helminth infections are necessary to dissect the causality of these relationships (cf. Cortés et al. 2018). Importantly, minimising variations between studies is crucial to warrant meaningful comparisons between datasets.

Whilst reducing the variability amongst samples collected from naturally helminth-infected humans may be difficult to achieve, the enormous impact that differences in technical and experimental approaches (from sample collection to bioinformatics and biostatistical analyses) exert on the overall variation detected across studies can be reduced (Figs. 1 and 2; Lindgreen et al. 2017; Costea et al. 2017; Golob et al. 2017). In particular, a range of bioinformatics pipelines are available for the analysis of high-throughput amplicon and metagenomics sequence datasets that include, e.g., different sequence-processing tools and reference databases for sequence annotation that could yield slightly different results (Lindgreen et al. 2017; Golob et al. 2017). For instance, the use of validated open microbiome analysis packages such Multiplexed Analysis of Projections by Sequencing (MAPseq) (Matias Rodrigues et al. 2017) or QIIME2 (https://qiime2.org/) may assist accurate taxonomic classifications of bacterial 16S rRNA amplicon datasets; similarly, sequence annotation should rely on the use of regularly updated reference databases. Amongst these, SILVA (https:/www.arb-silva.de/) (Quast et al. 2013) enables sensitive annotations of bacterial rRNA sequence data (Almeida et al.2018). The use of 
371 such standardized analysis workflows and reference databases for sequence annotation might

372 prove extremely useful to increase consistency across studies and enable researchers to identify

373 common and/or unique features between the gut microbiota of different host-parasite systems

374 which, in turn, might assist to better understand the mechanisms that regulate helminth-microbiota

375 relationships.

376 The consequences that elucidating such mechanisms may exert on future strategies of parasite 377 control are two-fold. First, disentangling the potential contribution of the gut flora to the pathogenesis of the infection is necessary in order to discover and develop new strategies to contrast helminth-associated pathology. Second, understanding the microbiota-dependent mechanisms by which parasitic helminths are able to modulate host immune responses and suppress inflammation may assist the discovery of novel immune-regulatory therapeutics against chronic inflammatory disorders of the GI tract that may act in synergy with helminth-based therapy (see Peachey et al. 2017 and Rapin and Harris, 2018 for reviews). However, in order for this new knowledge to be fully exploited in translational research, further studies that thoroughly consider inclusion/exclusion criteria for the selection of participants, include appropriate controls, and follow standardised experimental and data analysis protocols are necessary, and will allow to disentangle the potential influence of parasite-, drug- and/or population-dependent variables in each setting (Fig. 2). 


\section{ACKNOWLEDGEMENTS}

392 The authors would like to thank Professor R. Stephen Phillips for helpful suggestions on the draft 393 manuscript.

394 FINANCIAL SUPPORT

395 AC is supported by a postdoctoral fellowship from Fundación Alfonso Martín Escudero (Madrid, 396 Spain). LEP is supported by funding from the Horserace Betting Levy Board (HBLB) and TPJ 397 by scholarships by the Biotechnology and Biological Sciences Research Council (BBSRC) of the 398 United Kingdom. Research in the CC laboratory is supported by grants by the Royal Society and 399 the Isaac Newton Trust.

400 
Almeida A, Mitchell AL, Tarkowska A and Finn RD (2018) Benchmarking taxonomic assignments based on 16S rRNA gene profiling of the microbiota from commonly sampled environments. GigaScience 7, 1-10.

Barbour AD and Kafetzaki M (1991) Modeling the overdispersion of parasite loads. Mathematical Biosciences 107, 249-253.

Broadhurst MJ, Ardeshir A, Kanwar B, Mirpuri J, Gundra UM, Leung JM, Wiens KE, inflammatory signature and mucosal microbiota of the colon. PLoS Pathogens $\mathbf{8}$, e1003000.

Brosschot TP and Reynolds LA (2018) The impact of a helminth-modified microbiome on host immunity. Mucosal Immunology 11, 1039-1046. Tailoring water, sanitation, and hygiene (WASH) targets for soil-transmitted helminthiasis and schistosomiasis control. Trends in Parasitology 34, 53-63. Mitreva M, Krause L and Loukas A (2014) Impact of experimental hookworm infection on the human gut microbiota. The Journal of Infectious Diseases 210, 1431-1434. and Mitchell S (2016) Impact of helminth infections and nutritional constraints on the small intestine microbiota. PLoS One 11, e 0159770.

Chabé M, Lokmer A and Segurel L (2017) Gut protozoa: friends or foes of the human gut microbiota? Trends in Parasitology 33, 925-934.

Churcher TS, Ferguson NM and Basáñez MG (2005) Density dependence and overdispersion in the transmission of helminth parasites. Parasitology 131(Pt 1), 121-132. 
Cliffe LJ and Grencis RK (2004) The Trichuris muris system: a paradigm of resistance and susceptibility to intestinal nematode infection. Advances in Parasitology 57, 255-307. human infections with the whipworm, Trichuris trichiura, are not associated with alterations in the faecal microbiota. PLoS One 8, e76573.

Cortés A, Toledo R and Cantacessi C (2018) Classic models for new perspectives: delving into helminth-microbiota-immune system interactions. Trends in Parasitology 34, 640-654. Vlieg J, Junick J, Klymiuk I, Langella P, Le Chatelier E, Mai V, Manichanh C, Martin JC, Mery C, Morita H, O'Toole PW, Orvain C, Patil KR, Penders J, Persson S, Pons N, Popova M, Salonen A, Saulnier D, Scott KP, Singh B, Slezak K, Veiga P, Versalovic J, Zhao L, Zoetendal EG, Ehrlich SD, Dore J and Bork P (2017) Towards standards for human fecal sample processing in metagenomic studies. Nature Biotechnology 35, 1069-1076.

De Nardi R, Marchesini G, Li S, Khafipour E, Plaizier KJ, Gianesella M, Ricci R, Andrighetto I and Segato S (2016) Metagenomic analysis of rumen microbial population in dairy heifers fed a high grain diet supplemented with dicarboxylic acids or polyphenols. $B M C$ Veterinary Research 12, 29-016-0653-4.

Duarte AM, Jenkins TP, Latrofa MS, Giannelli A, Papadopoulos E, de Carvalho LM, Nolan MJ, Otranto D and Cantacessi C (2016) Helminth infections and gut microbiota - a feline perspective. Parasites \& Vectors 9, 625-016-1908-4.

Giacomin P, Zakrzewski M, Croese J, Su X, Sotillo J, McCann L, Navarro S, Mitreva M, Krause L, Loukas A and Cantacessi C (2015) Experimental hookworm infection and escalating 
451 gluten challenges are associated with increased microbial richness in celiac subjects. Scientific 452 Reports 5, 13797.

453

454

455

456

457

458

459

460

461

462

463

464

465

466

467

468

469

470

471

472

473

474

475

Giacomin P, Zakrzewski M, Jenkins TP, Su X, Al-Hallaf R, Croese J, de Vries S, Grant A, Mitreva M, Loukas A, Krause L and Cantacessi C (2016) Changes in duodenal tissueassociated microbiota following hookworm infection and consecutive gluten challenges in humans with coeliac disease. Scientific Reports 6, 36797.

Glendinning L, Nausch N, Free A, Taylor DW and Mutapi F (2014) The microbiota and helminths: sharing the same niche in the human host. Parasitology 141, 1255-1271.

Golob JL, Margolis E, Hoffman NG and Fredricks DN (2017) Evaluating the accuracy of amplicon-based microbiome computational pipelines on simulated human gut microbial communities. BMC Bioinformatics 18, 283-017-1690-0.

Greetham HL, Gibson GR, Giffard C, Hippe H, Merkhoffer B, Steiner U, Falsen E and Collins MD (2004) Allobaculum stercoricanis gen. nov., sp. nov., isolated from canine feces. Anaerobe 10, 301-307.

Holm JB, Sorobetea D, Kiilerich P, Ramayo-Caldas Y, Estelle J, Ma T, Madsen L, Kristiansen K and Svensson-Frej M(2015) Chronic Trichuris muris infection decreases diversity of the intestinal microbiota and concomitantly increases the abundance of Lactobacilli. PLoS One 10, e0125495.

Hotez PJ, Alvarado M, Basanez MG, Bolliger I, Bourne R, Boussinesq M, Brooker SJ, Brown AS, Buckle G, Budke CM, Carabin H, Coffeng LE, Fevre EM, Furst T, Halasa YA, Jasrasaria R, Johns NE, Keiser J, King CH, Lozano R, Murdoch ME, O'Hanlon S, Pion SD, Pullan RL, Ramaiah KD, Roberts T, Shepard DS, Smith JL, Stolk WA, Undurraga EA, Utzinger J, Wang M, Murray CJ and Naghavi M (2014) The global burden of disease study 2010: interpretation and implications for the neglected tropical diseases. PLoS Neglected Tropical Diseases 8, e2865. 
IS (2015) Chronic Trichuris muris infection in C57BL/6 mice causes significant changes in host microbiota and metabolome: effects reversed by pathogen clearance. PLoS One 10, e0125945. microbiome and metabolome of Strongyloides stercoralis infected volunteers from a nonendemic area. Scientific Reports 8, 15651.

Jenkins TP, Peachey LE, Ajami NJ, MacDonald AS, Hsieh MH, Brindley PJ, Cantacessi C and Rinaldi G (2018a) Schistosoma mansoni infection is associated with quantitative and qualitative modifications of the mammalian intestinal microbiota. Scientific Reports 8, 12072.

Jenkins TP, Rathnayaka Y, Perera PK, Peachey LE, Nolan MJ, Krause L, Rajakaruna RS and Cantacessi C (2017) Infections by human gastrointestinal helminths are associated with changes in faecal microbiota diversity and composition. PLoS One 12, e0184719.

Kay GL, Millard A, Sergeant MJ, Midzi N, Gwisai R, Mduluza T, Ivens A, Nausch N, Mutapi F and Pallen M(2015) Differences in the faecal microbiome in Schistosoma haematobium infected children vs. uninfected children. PLoS Neglected Tropical Diseases 9, e0003861.

Kelly BJ, Gross R, Bittinger K, Sherrill-Mix S, Lewis JD, Collman RG, Bushman FD and

Li H (2015) Power and sample-size estimation for microbiome studies using pairwise distances and PERMANOVA. Bioinformatics 31, 2461-2468.

Kim JY, Kim EM, Yi MH, Lee J, Lee S, Hwang Y, Yong D, Sohn WM and Yong TS (2018) Intestinal fluke Metagonimus yokogawai infection increases probiotic Lactobacillus in mouse cecum. Experimental Parasitology 193, 45-50. diversity of the gut microbiota. PLoS Neglected Tropical Diseases 8, e2880. 
502

503

504

505

506

507

508

509

510

511

512

513

514

515

516

517

518

519

520

521

522

523

524

525

526

Leung JM, Graham AL and Knowles SCL (2018) Parasite-microbiota interactions with the vertebrate gut: synthesis through an ecological lens. Frontiers in Microbiology 9, 843.

Ley RE, Hamady M, Lozupone C, Turnbaugh PJ, Ramey RR, Bircher JS, Schlegel ML, Tucker TA, Schrenzel MD, Knight R and Gordon JI (2008) Evolution of mammals and their gut microbes. Science 320, 1647-1651.

Li RW, Li W, Sun J, Yu P, Baldwin RL and Urban JF (2016) The effect of helminth infection on the microbial composition and structure of the caprine abomasal microbiome. Scientific Reports 6, 20606.

Lindgreen S, Adair KL and Gardner PP (2016) An evaluation of the accuracy and speed of metagenome analysis tools. Scientific Reports 6, 19233.

Lozupone CA, Stombaugh JI, Gordon JI, Jansson JK and Knight R (2012) Diversity, stability and resilience of the human gut microbiota. Nature 489, 220-230.

Martin I, Djuardi Y, Sartono E, Rosa BA, Supali T, Mitreva M, Houwing-Duistermaat JJ and Yazdanbakhsh M (2018) Dynamic changes in human-gut microbiome in relation to a placebo-controlled anthelminthic trial in Indonesia. PLoS Neglected Tropical Diseases 12, e0006620.

Mutapi F (2015) The gut microbiome in the helminth infected host. Trends in Parasitology 31, 405-406.

Matias Rodrigues JF, Schmidt TSB, Tackmann J and von Mering C (2017) MAPseq: highly efficient k-mer search with confidence estimates, for rRNA sequence analysis. Bioinformatics 33, $3808-3810$.

O'Connell EM and Nutman TB (2016) Molecular diagnostics for soil-transmitted helminths. The American Journal of Tropical Medicine and Hygiene 95, 508-513.

Peachey LE, Jenkins TP and Cantacessi C (2017) This gut ain't big enough for both of us. Or is it? Helminth-microbiota interactions in veterinary species. Trends in Parasitology 33, 619-632. 

Cantacessi C (2018) The relationships between faecal egg counts and gut microbial composition in UK thoroughbreds infected by cyathostomins. International Journal for Parasitology 48, 403412.

Pearce EJ and MacDonald AS (2002). The immunobiology of schistosomiasis. Nature Reviews Immunology 2, 499-511.

Pérez-Muñoz ME, Bergstrom K, Peng V, Schmaltz R, Jiménez-Cardona R, Marsteller N, McGee S, Clavel T, Ley R, Fu J, Xia L and Peterson DA (2014) Discordance between changes in the gut microbiota and pathogenicity in a mouse model of spontaneous colitis. Gut Microbes $\mathbf{5}$, 286-295. (2013) The SILVA ribosomal RNA gene database project: improved data processing and webbased tools. Nucleic Acids Research 41, 590-596.

540 Ramanan D, Bowcutt R, Lee SC, Tang MS, Kurtz ZD, Ding Y, Honda K, Gause WC, Blaser

541 MJ, Bonneau RA, Lim YA, Loke P and Cadwell K (2016) Helminth infection promotes 542 colonization resistance via type 2 immunity. Science 352, 608-612.

543 Rapin A and Harris NL (2018) Helminth-bacterial interactions: cause and consequence. Trends 544 in Immunology 39, 724-733. Small intestinal nematode infection of mice is associated with increased enterobacterial loads alongside the intestinal tract. PLoS One 8, e74026. MJ, Finlay BB and Maizels RM (2014) Commensal-pathogen interactions in the intestinal tract: Lactobacilli promote infection with, and are promoted by, helminth parasites. Gut Microbes $\mathbf{5}$, $522-532$.

Rosa BA, Supali T, Gankpala L, Djuardi Y, Sartono E, Zhou Y, Fischer K, Martin J, Tyagi R, Bolay FK, Fischer PU, Yazdanbakhsh M and Mitreva M (2018) Differential human gut microbiome assemblages during soil-transmitted helminth infections in Indonesia and Liberia. Microbiome 6, 33-018-0416-5. 

Keiser J (2018a) Investigations on the interplays between Schistosoma mansoni, praziquantel and the gut microbiome. Parasites \& Vectors 11, 168-018-2739-2.

Sekirov I, Russell SL, Antunes LC and Finlay BB (2010) Gut microbiota in health and disease. Physiological Reviews 90, 859-904.

Stensvold CR and van der Giezen M (2018) Associations between gut microbiota and common luminal intestinal parasites. Trends in Parasitology 34, 369-377.

Su C, Su L, Li Y, Long SR, Chang J, Zhang W, Walker WA, Xavier RJ, Cherayil BJ and Shi HN (2018) Helminth-induced alterations of the gut microbiota exacerbate bacterial colitis. Mucosal Immunology 11, 144-157.

Wang J, Tang H, Zhang C, Zhao Y, Derrien M, Rocher E, van-Hylckama Vlieg JE, Strissel K, Zhao L, Obin M and Shen J (2015) Modulation of gut microbiota during probiotic-mediated attenuation of metabolic syndrome in high fat diet-fed mice. The ISME Journal 9, 1-15.

573 Wang WL, Xu SY, Ren ZG, Tao L, Jiang JW and Zheng SS (2015) Application of metagenomics in the human gut microbiome. World Journal of Gastroenterology 21, 803-814.

World Health Organisation (2006) Preventive chemotherapy in human helminthiasis: coordinated use of anthelminthic drugs in control interventions: a manual for health professionals and programme managers. Geneva: WHO; 2006.

Wu S, Li RW, Li W, Beshah E, Dawson HD and Urban JF Jr. (2012) Worm burden-dependent disruption of the porcine colon microbiota by Trichuris suis infection. PLoS One 7, e35470. 


\section{FIGURE LEGENDS}

587 Fig. 1 Sources of variation and confounding factors potentially impacting the outcome of studies 588 of human-helminth-gut microbiota interactions in helminth-endemic regions.

589 Fig. 2 Proposed approaches aimed at reducing the methodological sources of variation 590 surrounding investigations of human-helminth-gut microbiota interactions. 


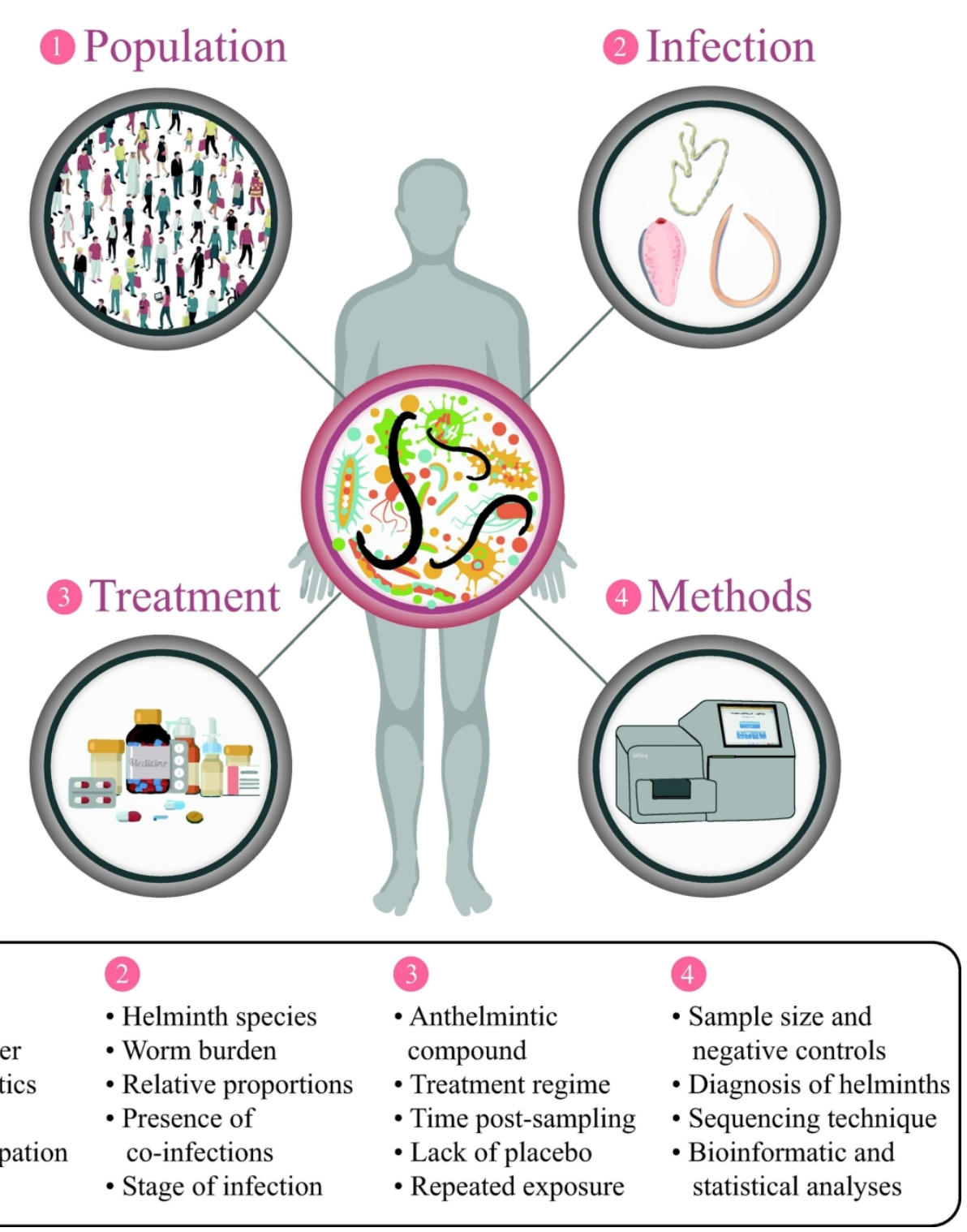

Figure 1 . Sources of variation and confounding factors potentially impacting the outcome of studies of human-helminth-gut microbiota interactions in helminth-endemic regions.

$158 \times 180 \mathrm{~mm}(300 \times 300$ DPI $)$ 
(1) Population

- Balanced gender ratios

- Delimited age ranges

- Homogeneous socioeconomic status of study subjects

- Lifelong residence in the study area

- Comparison of similar populations across different geographical locations
2 Infection

- Diagnostic screening of intestinal and extra-intestinal bacterial, viral, and/or protozoan pathogens

- Estimation of infection intensities according to standardised scales (WHO 2006)

- Absence of clinical symptoms of gut and/or systemic diseases
- History of recent anthelmintic and/or antibiotic treatment

- Inclusion of placebo-treated groups

- Assessment of potential effects of treatment on the gut microbiota

- Verification of infection clearance following anthelmintic treatment

- Multiple samplings post-treatment (longitudinal assessment of variations)
- Suitable diagnostic tools for pathogen identification

- Sample size and statistical power calculation

- Standardised protocols for sample collection, storage, and nucleic acid extraction

- Standardised pipelines for bioinformatic and statistical analysis of sequence data

\section{Treatment}

\section{Methods}

Figure 2. Proposed approaches aimed at reducing the methodological sources of variation surrounding investigations of human-helminth-gut microbiota interactions.

$163 \times 164 \mathrm{~mm}(300 \times 300$ DPI $)$ 
2

3

4

5

6

7

8

9

10

11

12

13

14

15

16

17

18

19

20

21

22

23

24

25

26

27

28

Invited review

\section{Helminths and microbes within the vertebrate gut - not all studies are created equal

\begin{abstract}
Alba Cortés ${ }^{1}$, Laura E. Peachey ${ }^{1,2}$, Timothy P. Jenkins ${ }^{1}$, Riccardo Scotti $^{1}$ and Cinzia Cantacessi ${ }^{1}$
${ }^{1}$ Department of Veterinary Medicine, University of Cambridge, Madingley Road CB3 OES, Cambridge, United Kingdom
${ }^{2}$ Bristol Veterinary School, Faculty of Health Sciences, University of Bristol, Langford House, Langford, BS40 5DU, Bristol, United Kingdom

Running title: Helminth-microbiota interactions in the human gut

\section{Corresponding author:}

Cinzia Cantacessi

Department of Veterinary Medicine, University of Cambridge, Madingley Road CB3 OES, Cambridge, United Kingdom

Tel. +44 (0) 1223760541

Fax. +44 (0)1223 337610

E-mail: cc779@cam.ac.uk

4, $\underline{648421}$ words 
29 SUMMARY

30 The multifaceted interactions occurring between gastrointestinal (GI) parasitic helminths and the 31 host gut microbiota are emerging as a key area of study within the broader research domain of 32 host-pathogen relationships. Over the past few years, a wealth of investigations has demonstrated 33 that GI helminths interact with the host gut flora, and that such interactions result in modifications 34 of the host immune and metabolic statuses. Nevertheless, whilst selected changes in gut microbial 35 composition are consistently observed in response to GI helminth infections across several host36 parasite systems, research in this area to date is largely characterised by inconsistent findings. 37 These discrepancies are particularly evident when data from studies of GI helminth-microbiota 38 interactions conducted in humans from parasite-endemic regions are compared. In this review, 39 we provide an overview of the main sources of variance that affect investigations on human40 helminth-gut microbiota interactions and propose a series of methodological approaches that, 41 whilst accounting for aking into account the inevitable constraints of human fieldwork, are aimed 42 at minimising confounding factors and draw biologically meaningful interpretations from highly 43 variable datasets. 
A plethora of experimental evidence supports a key role of infections by gastrointestinal (GI) helminth parasites in shaping the composition of the vertebrate gut microbiota, with significant implications for local and systemic host immunity (reviewed by Brosschot and Reynolds, 2018). For instance, recent studies have partly attributed the parasite-associated qualitative and/or quantitative alterations to host GI microbial profiles to the ability of GI helminths to stimulate the initiate-initial the-onset of T-regulatory (Treg) immune mechanismsresponses, which result in down-regulation of inflammatory responses and establishment of chronic infections, to helminthparasite-associated qualitative and/or quantitative alterations to GI microbial profiles the ability to initiate the onset of T-regulatory (Treg) immune mechanisms, that result in downregulation of inflammatory responses and establishment of chronic infections (cf. Cantacessi et al. 2014; Reynolds et al. 2014; Giacomin et al. 2015, 2016; Zaiss et al. 2016). On the other hand, other studies have reported associations between acute helminth infections and gut mierobiome $\underline{\text { microbiota imbalances }(=\text { dysbiosis }) \text { characterised by that involve-significant expansion of }}$ populations of putative pro-inflammatory bacteria (e.g. Rausch et al. 2013; Jenkins et al. 2018a;

Schneeberger et al. 2018a); these observations have, thus lending-lent credit to the hypothesis that

helminth-associated alterations of gut microbiota compositionme may lead to both localised and systemic consequences for the host organism, that includeing immunopathology and (e.g. Rausch at. 2013; Jenkins al. 2018; Schneeberger al. 2018a) 2 and as well as exacerbated malnutrition in at-risk subjects from parasite-endemic areas (reviewed by Glendinning et al. 2014; Houlden et al. 2015; Cattadori et al. 2016).

Over the past decade, newly acquired knowledge of the impact that GI helminth infections exert on the vertebrate gut microbialeme composition and metabolism has contributed to a better understanding of parasite systems biology and host-pathogen interactions (reviewed by Peachey et al. 2017; Leung et al. 2018; Rapin and Harris et al. 2018), and has been proposed as a first step towards the identification and development of novel strategies of parasite control based on the targeted manipulation of the host gut microbiota (cf. Peachey et al. 2017). Nevertheless, for humans in particular, progress in this field of research is greatly impaired by the impact of several 
confounding factors that inevitably affect studies conducted in naturally infected individuals (Mutapi, 2015; Chabé et al. 2017). In this review, we summarise current knowledge of GI helminth-microbiome interactions in humans under natural conditions of infection, identify similarities and differences between datasets and provide an overview of the confounding factors that may affect the interpretation of findings.

\section{HUMAN-HELMINTH-GUT MICROBIOTA INTERACTIONS IN REAL-WORLD}

\section{SCENARIOS}

In endemic areas for helminthiases, the vast majority of infected individuals harbour multiple helminth species, often occupying different niches of the host organism (Hotez et al. 2010). Whilst polyparasitism is often regarded as a major confounding factor in investigations of parasite-microbiota interactions conducted in humans under natural conditions of infection (Cooper et al. 2013; Jenkins et al. 2017; Martin et al. 2018; Rosa et al. 2018), findings from these studies are key to assessing the impact that GI helminths exert on gut microbiota homeostasis in a 'real-world' scenario. Nevertheless, several factors should be considered when interpreting results obtained from individuals infected by multiple helminth species. First, anthropometric (e.g. age and gender) and anthropologic variables (e.g. ethnicity, diet and occupation) are well known to profoundly impact the 'baseline' composition of the human gut microbiotame (Sekirov et al. 2010; Yatsunenko et al. 2012) (cf. Fig. 1); therefore, the enrolment of large cohorts of individuals is often necessary in order to achieve sufficient statistical power and avoid uninformative and/or misleading results (Kelly et al. 2015). However, in many studies, the number of individuals enrolled and samples analysed is inevitably dictated by logistical and financial constraints. In these instances, population-related variables that impact gut microbiota composition may contribute substantially to inconsistencies among findings from different studies (cf. Fig. 1). For instance, a negative association between colonisation by the whipworm Trichuris trichiura and the abundance of bacteria belonging to the genus Prevotella in the faeces of infected individuals has been reported in two separate studies conducted in Malaysia (Lee et al. 2014; Ranaman et al. 2016), while other studies conducted in Ecuador, and Liberia and Indonesia, 
respectively, have failed to identify significant variations in faecal populations of Prevotella in individuals either solely infected by T. trichiura or co-infected with other species of soiltransmitted helminths (STHs) (Cooper et al. 2013; Martin et al. 2015; Rosa et al. 2018).

In addition, whilst Rosa and co-authors (2018) detected several distinctive features in the gut microbial profiles of helminth-harbouring individuals that were specifically associated to single infections with the hookworm Necator americanus, the roundworm Ascaris lumbricoides or $T$. trichiura, such features were inconsistent between two independent cohorts of helminth-infected volunteers from Liberia and Indonesia, respectively; this discrepancy suggests that other yet undetermined environmental factors may contribute to qualitative and quantitative alterations of the gut microbial profiles of helminth-infected individuals from different geographical areas. In contrast, an association between the abundance of selected bacterial taxa and infections by one or more STHs could be consistently detected in samples from both Liberian and Indonesian cohorts (Rosa et al. 2018). These taxa included bacteria belonging to the genera Olsenella and Allobaculum, which were expanded in the gut microbiota of helminth-infected individuals when compared to that of uninfected controls. To the best of our knowledge, the study by Rosa et al. (2018) was the first to report a link between infections by STHs and the abundance of these bacterial genera in the human gut. Interestingly, in mice suffering from metabolic syndrome, administration of probiotics was followed by expansion of populations of Olsenella and/or Allobaculum, and a reduction in systemic and/or local gut inflammatory responses (Wang et al. 2015). Moreover, Allobaculum spp. are putative producers of anti-inflammatory short-chain fatty acids (Greetham et al. 2004), and are severely reduced in the gut of mice genetically predisposed to spontaneous colitis (Pérez-Muñoz et al. 2014). This knowledge led Rosa et al. (2018) to hypothesize that these bacteria may play a yet undetermined role in the anti-inflammatory properties of parasitic helminths, and reinforce the proposition that the interactions between thus underpinning the general idea that hosts, -parasites and -gut microbiota are interactions are 
127 negative association between the genus Allobaculum and colonisation by GI helminths has been 128 observed in a mouse model of chronic trichuriasismice chronically infected with $T$. muris (Holm 129 et al. 2015), in which is featured by a dominant Th1-mediated immune responses are dominant 130 (reviewed by Cliffe and Grencis, 2004), as well as in mice-and with patent infection by the blood

131 fluke Schistosoma mansoni (Jenkins et al. 2018a), in which migrating eggs are responsible for the onset of marked Th2-mediated inflammatory responses-are elicited to migrating eggs (reviewed

133 by Pearce and MacDonald, 2002). The immune-molecular mechanisms through-via which 134 members of the genus Allobaculum may regulate local and systemic inflammation are to be elucidatedstill unclear (Greetham et al. 2004; Pérez-Muñoz et al. 2014; Wang et al. 2015).

Nonetheless, current data experimental evidence on-showing concomitant-reductions in populations of Allobaculum andalongside helminth-associated gut inflammation supports seems consistent with-the hypothesis of -raised by Rosa et al. (2018); in the future,-, suggesting that

139 taboratery rodent models of GI helminthiasis-helminth infections whose gut microbiota is 140 deprived of, and subsequently recolonised with, the genus Allobaculum could be exploited to investigate the potential involvement of these bacteria in the parasite-mediated

142 immunomodulation-mediated by helminth parasites (e.g. via exogenous recolonization with Allobacultm spp.). Notably, both models of helminth infection are characterised by the oceurrence of severe intestinal inflammation involving different populations of $\mathrm{T}$ CD4+ cells (i.e. Th1 and Th2, respectively; Pearce and MacDonald, 2002; Cliffe and Grencis, 2004), and therefore, the observed reduction in populations of Allobaculum in these systems supports the immune

147 regulatory role for this bacterial genus.

148 Beside the intrinsic variability of the human gut microbiota, studies conducted under natural conditions of helminth colonisation are likely to be affected by factors linked to the different combinations of infecting species and their relative abundances. For instance, in a study conducted in a cohort of Ecuadorian children, the specific features detected in the gut microbial profiles of subjects co-infected with T. trichiura and A. lumbricoides could not be identified in the microbiota of Trichuris-only infected individuals (Cooper et al. 2013). Similarly, selected 
microbial features that were observed in studies conducted in human volunteers with monospecific infections with, for instance, A. lumbricoides, could not be detected in the gut microbiota of subjects harbouring the same parasite alongside other helminth species (e.g. T. trichiura and N. americanus) (Rosa et al. 2018), thus suggesting that a complex interplay exists between the host gut and its macro- and microbiota, that might be difficult to replicate in experimental settings. Furthermore, current evidence obtained from animal models of helminth infections indicates that worm burdens can impact the nature and/or the magnitude of parasite-associated alterations in gut microbial composition (Wu et al. 2012; Peachey et al. 2018) $;$ neverthelessNevertheless, such evidence is not yet available for human infections, in which whose burdens-parasite burdens in endemic areas-may range from low to very high due to overdispersion of parasite loads in endemic areas (Barbour and Kafetzaki, 1991; Churcher et al. 2005)-and, therefore, are likely to be an important confounding factor for studies of parasite-microbiota interactions in naturally infected individuals.

Another frequent constraint of investigations conducted in cohorts of human subjects with natural helminth infections is the limited availability of 'genuine' negative controls, i.e. individuals from the same communities of parasite-infected subjects who lack previous exposure to infections by parasitic helminths. Instead, individuals with no evidence of patent helminth infections are inevitably enrolled as control subjects (e.g. Cooper et al. 2013; Lee et al. 2014; Jenkins et al. 2017; Rosa et al. 2018); nevertheless, studies in helminth-infected individuals subjected to anthelmintic treatment, as well as in primates and pigs exposed to Trichuris spp., have shown that between the gut microbial profiles of helminth-infected and uninfected volunteers from the same communities. In addition, patent infections are often diagnosed using stool-based microscopic methods, that are known for their relatively low sensitivity and that may yield false negative results, e.g. in case of intermittent shedding of eggs and/or larvae (O'Connell and Nutman, 2016). 
181 Recently, Rosa et al. (2018) used quantitative real-time PCR to diagnose STH infections in 182 individuals subjected to gut microbiotame profiling, indicating that this technique may represent 183 a robust and sensitive alternative to microscopic methods, since it provides users with the ability to semi-quantify burdens of different helminth species from minute amounts of DNA template. However, in spite of their higher sensitivity, molecular methods rely on the use of primers that selectively target the parasite species of interest, thus impairing the simultaneous detection of potential (asymptomatic or subclinical) co-infections with other helminth and/or non-helminth pathogens (O'Connell and Nutman, 2016). Indeed, the impact of protozoa on the gut microbial diversity and composition has been clearly demonstrated in humans and other vertebrates (reviewed by Chabé et al. 2017; Stensvold and van der Giezen, 2018). Furthermore, a recent study conducted in a cohort of Colombian schoolchildren reported common features in the faecal microbial composition of subjects co-infected with helminths and protozoans and monoparasitized with the flagellate Giardia intestinalis compared to uninfected individuals (ToroLondono et al. 2019). Whilst the mechanisms via which each group of parasites alters the host gut flora, as well as the nature of such alterations, are yet to be determined, these findings support the need to conduct additional diagnostic tests on stool samples from helminth-infected cohorts, as well as the corresponding uninfected subjects, in order to rule out the influence of concomitant bacterial, viral and/or protozoan infections that may be responsible for the changing gut microbial profiles of these individuals (cf. Chabé et al. 2017).

Nevertheless, in spite of the several confounding factors outlined above (cf. Fig. 1), observational studies in helminth endemic areas have proven useful for the identification of significant associations between parasite colonisation and the gut microbial profiles of humans under natural conditions of infection. Importantly, studies conducted in these communities provide excellent opportunities to evaluate the effect(s) that parasite removal (e.g. via the administration of broadspectrum anthelmintics) exert(s) on the gut microbiota of previously infected individuals, thus contributing cues to understand the causality of helminth-microbiota relationships. 
The implementation of mass drug administration programmes in endemic areas for STHs and schistosomiasis offers opportunities to elucidate potential mechanisms via which parasitic helminths modulate the host gut microbiota. For instance, qualitative and quantitative changes in gut microbial profiles that are caused by direct interactions between parasites and gut bacteria may be expected to rapidly reverse following parasite removal, whilst long-lasting alterations are likely to result from indirect interplay mediated by the host immune system (Houlden et al. 2015; Su et al. 2018). Nevertheless, such investigations are also generally constrained by the presence of several confounding factors that include not only the host- and parasite-dependent variables outlined above, but also variations linked to the use of different drugs and treatment regimes (Schneeberger et al. 2018b), as well as time of sampling post-anthelmintic treatment (Houlden et al. 2015) (Fig. 1). The latter in particular may profoundly affect findings from these studies, as the presence of tissue lesions caused by e.g. parasite feeding activity and location (e.g. bloodfeeders $v s$. non blood-feeders and luminal $v s$. tissue dwellers) are likely to influence the timespan between helminth removal and microbiome recovery (reviewed by Leung et al. 2018). Moreover, for ethical reasons, data from these experiments is often biased by the lack of placebo-treated control groups. These limitations may be at least partially responsible for the differences between findings from studies aimed to elucidateat elucidating the effect of deworming on the gut microbiota of helminth-infected volunteers; notwithstanding, it is worth noting that, in instances where deworming-associated changes in human gut microbial profiles were detected, these were generally moderate (Ramanan et al. 2016; Martin et al. 2018; Schneeberger et al. 2018b).

Consistent with this, a recent study conducted on faecal samples collected from a rural community in Indonesia reported that the composition of the gut microbiotame of individuals repeatedly treated with either albendazole or placebo (for 21 months) resembled that of samples collected from the same subjects prior to treatment, rather than that of uninfected controls (Rosa et al. 2018). Moreover, a parallel investigation conducted on the same cohort of individuals detected reduced populations of Prevotella in albendazole-treated subjects in which complete deworming did not occur, compared to placebo-treated individuals with patent helminth infections (Martin et 
al. 2018). Intriguingly, failure of albendazole treatment was accompanied by a dominance of $T$. trichiura (over other helminth species) in these subjects, while placebo-treated individuals maintained a diverse macrobiota (i.e. multiple helminth infections); hence, differences in the composition of the GI macrobiota (i.e. species present and their relative abundances) between albendazole- and placebo-treated individuals could account for variations in the composition of the intestinal microflora of these subjects (Martin et al. 2018). Significant associations between colonisation by T. trichiura and Prevotella abundance were not observed in the Indonesian cohort (Martin et al. 2018; Rosa et al. 2018). However, negative associations between whipworm infections and Prevotella abundance had been detected previously in two independent studies conducted in Malaysia (Lee et al. 2014; Ramanan et al. 2016). In particular, Ramanan and coauthors (2016) observed that, following albendazole treatment, expansion of Prevotella populations in the human faecal microbiota was related to reduced T. trichiura faecal egg counts. In contrast, no significant associations between helminth infection and abundance of bacteria belonging to the genus Prevotella was reported in a study investigating the impact of parasite colonisation and effective-successful treatment with a combination of albendazole and ivermectin treatment on the faecal microbial profiles of a cohort of Trichuris-infected children from Ecuador (Cooper et al. 2013), nor in a group of helminth-infected adults from Sri Lanka treated with pyrantel pamoate (Jenkins et al. 2017). Similarly, no qualitative or quantitative changes to faecal microbial composition were observed in two cohorts of schoolchildren from Côte d'Ivoire and Zimbabwe infected by S. mansoni and S. haematobium, respectively, following treatment with praziquantel (Kay et al. 2014; Schneeberger et al. 2018a). However, successful elimination of $S$. mansoni was associated with a higher abundance of Fusobacterium spp. pre-treatment, as well as $24 \mathrm{hrs}$ post-treatment (Schneeberger et al. 2018a).

Whilst drug administration in endemic regions may result in effective elimination of helminth infections, potential co-infecting protozoan parasites are not susceptible to anthelmintic treatment; this, together with the sub-standard hygienic and sanitary conditions that generally characterise these areas and that result in continuous re-exposure to infective helminth 
262 developmental stages (Campbell et al. 2018), impairs the full assessment of the consequences of 263 helminth removal on the composition of the human gut microbiota. To the best of our knowledge, 264 thus far, a single study has investigated the effects of chronic infections by a GI helminth, 265 Strongyloides stercoralis, and anthelmintic treatment on the composition of the faecal 266 microbiotame and metabolome of humans from a non-endemic area of Europe, where parasite transmission had been interrupted (Jenkins et al. 2018b). Treatment with ivermectin resulted in compositional changes of the faecal microbiota (analysed 6 months post-treatment), which partially resembled that of uninfected control subjects (Jenkins et al. 2018b); in particular, alpha diversity [ $=$ a measure of the number of bacterial species present in a given microbial community (richness) and their relative abundance (evenness)] was reduced in the microbiota of the former group ofdewormedindividuals post-treatment (although statistical significance was not achieved) and accompanied by expanded populations of potentially pathogenic bacteria (Jenkins et al. 2018b). In addition, the faecal metabolic profiles obtained from samples collected post-ivermectin treatment shared features with both appeared to fall somewhere in between-those obtained from samples collected pre-treatment as well as fromand from uninfected controls_(Jenkins et al. 2018b); this observation led Jenkins et al. (2018b), to hypothesise that, thus supporting the notion that, following parasite removal and over time, suggesting a (direct and/or indirect) effect of parasite infection and removal on both gut microbiotame and metabolome may revert to the original a-pre-infection state. Multiple factors, including but not limited to those outlined above, may contribute to the discrepancies observed between the findings from this work and those that reported no or minor effects of anthelmintic treatment on the gut microbiome of helminth-infected humans (Cooper et al. 2013; Ramanan et al. 2016; Martin et al. 2018; Rosa et al. 2018; Schneeberger et al. 2018a,b).

Despite the limitations outlined above, studies of GI helminth-microbiota relationships conducted in endemic areas for helminthiases have provided repeated evidence of the perturbations that parasites and anthelmintic treatment exert on the equilibrium of resident populations of gut bacteria and on gut homeostasis. However, the identification of common signatures across studies 
remains key to designing future experiments, e.g. in animal models of helminth infections, that may assist the elucidation of the mechanisms that underpin the interactions between GI helminths, the gut microbiota and the host immune system.

\section{DO COMMON SIGNATURES EXIST ACROSS STUDIES OF HOST-HELMINTH-} MICROBIOTA INTERACTIONS?

The identification of gut microbial signatures that occur reproducibly across several host-GI helminth systems is crucial for designing novel anti-helminth intervention strategies based on the manipulation of the gut microbiota (Peachey et al. 2017). Studies conducted in animal models of helminth infections are expected to assist the identification of such signatures, as well as the direct (i.e. parasite-mediated) and/or indirect (i.e. immune-mediated) mechanisms that govern helminthmicrobiota interactions (Cortés et al. 2018); nevertheless, the inconsistencies that characterise studies of helminth-microbiota relationships published to date make such a task highly challenging. Indeed, for patterns to be identified, fluctuations in selected populations of gut microbes must be interpreted in light of the physical and immunological alterations of the mucosal environment in which such alterations occur (Leung et al. 2018). For instance, expanded populations of Lactobacillaceae have been repeatedly detected following infection with several species of parasitic helminths in several host species (Reynolds et al. 2014; Duarte et al. 2015; Holm et al. 2015; Houlden et al. 2015; Cattadori et al. 2016; Jenkins et al. 2018a; Kim et al. 2018), and could thus be considered as a 'consistent alteration' in gut microbiota composition upon helminth colonisation. However, key differences exist between host-parasite pairs investigated in the studies that have reported such an outcome. Indeed, whilst populations of Lactobacillaceae promote regulatory responses in mice infected by Heligmosomoides polygyrus bakeri (Reynolds et al. 2014), a lack of correlation between Lactobacillaceae abundance and Treg populations has been observed in other host-parasites systems, such as mice chronically infected with T. muris and rabbits infected with Trichostrongylus retortaeformis, in which the expansion of populations of gut Lactobacillaceae upon helminth infection occurs in an environment dominated by Th1-mediated immune responses (Holm et al. 2015; Houlden et al. 2015; Cattadori 
et al. 2016). These differences suggest that alternative mechanisms may regulate the differentiation and development of adaptive immune responses in each host-parasite system (Houlden et al. 2015), and thus that similar alterations in gut microbiota composition may result in different consequences that are dependent on the microenvironment where these changes occur. Notwithstanding, the interactions between hosts, helminths and the gut microbiota are likely multifaceted and multidirectional, and therefore the potential consequences that selected compositional changes in gut microbiota exert on host homeostasis are only one aspect of these complex interplay. For instance, a common yet undetermined mechanism may determine the expansion of Lactobacillaceae in the gut of helminth-infected hosts.

On the other hand, apparent 'contradictory' findings across studies may result from fundamental differences between gut compartments under investigation. For instance, Prevotella spp. was expanded in the abomasum and faeces of sheep infected by abomasal trichostrongyles (i.e. Haemonchus contortus and Teladorsagia circumcincta; Li et al. 2016; Cortés et al. in preparation), whilst the same taxa were reduced in the faeces of a range of host species, including mice, humans and horses, infected by nematodes residing in the large intestine, i.e. Trichuris spp. and cyathostomins, respectively (Lee et al. 2014; Houlden et al. 2015; Peachey et al. submitted). It must be noted, however, that whilst increased abomasal $\mathrm{pH}$ favours Prevotella overgrowth in the abomasum (De Nardi et al. 2016; Li et al. 2016), the same taxa are likely to be exposed to a dramatically different microenvironment in the large intestine that may determine the contraction of these bacterial taxagroups. In addition, given the functional dissimilarities between the abomasal and colonic microbiota, such alterations are expected to result in fundamentally different outcomes for the homeostasis of each of these gut compartments (Ley et al. 2008), and hence comparisons are, in our opinion, unwarranted.

In parallel to species of bacteria with functions that may vary depending on the gut compartment, multiple taxa share the same functions in different microenvironments (Lozupone et al. 2012); therefore, it is plausible that, even though inconsistencies are detected across studies, these may result in similar functional alterations in the host-parasite pairs being compared. For instance, 
recent studies in mouse and humans infected with $S$. mansoni have reported the expansion of different genera of bacteria with pro-inflammatory functions in the gut microbiota of the respective hosts (Jenkins et al. 2018a; Schneeberger et al. 2018a). These observations lend credit to the hypothesis that the functional role of the gut microbiota in helminth infections could be far less 'diverse' than the taxonomic associations reported thus far. For this hypothesis to be confirmed or confuted, a better understanding of the function(s) of each bacterial taxon inhabiting the different gut compartments in a range of host species is needed. To this aim, the integration of metagenomic, metabolomic and metatranscriptomic technologies, alongside traditional microbiology and microscopy techniques, may assist to achieve a holistic picture of the impact of GI helminth infections on the functions of the human gut microbiota, and its significance for disease pathophysiology and overall host health (Wang et al. 2015).

\section{CURRENT NEEDS AND FUTURE DIRECTIONS}

Understanding the complex interactions between GI helminths and their vertebrate hosts is pivotal for advancing our knowledge off the fundamental biology of these parasites and the diseases they cause (see Peachey et al. 2017; Leung et al. 2018; Rapin and Harris et al. 2018 for reviews). Whilst the role of the gut microbiota in host-parasite relationships has long been overlooked, current knowledge of the key roles that resident bacteria play in host health and disease, together with recent technical advancements for microbiota profiling, have boosted research is this area. This is currently leading to increasing evidence of an active involvement of the gut microbiota in the immunopathology of GI helminth infections (e.g. Rausch et al. 2013; Jenkins et al. 2018a; Schneeberger et al. 2018a). Furthermore, several studies support a role for the gut microbiota in the immune regulatory properties of helminth parasites (Cantacessi et al. 2014; Reynolds et al. 2014; Giacomin et al. 2015, 2016; Zaiss et al. 2016). FurthermoreIn addition, data collected to date points towards a likely role whether certain members-of the gut microflora in are actively involved in-the immunopathology of particular-selected GI helminth infections that awaits experimental validationis a currently outstanding question that awaits for a response. Indeed, whilst $t$ Trying to untangle the relevance of particular fluctuations of specific bacterial taxa on 
370 infection outcome is challenging; nevertheless,- currently available data suggest that low-

371 intensity, long-term helminth infections are commonly linked to high microbial diversity and 372 predominance of bacteria typically associated with gut health ${ }_{-\dot{\bar{\prime}}}$ Cenversely, high-intensity, acute 373 infections are often associated to gut dysbiosis, characterised by reduced alpha diversity and an 374 increase in pro-inflammatory and often opportunistic pathogens (Peachey et al. 2017). However, 375 for this knowledge to be exploited in translational studies, further investigations in both natural 376 and experimental settings are needed to distinguish spurious results from genuine helminth377 microbiota associations (Peachey et al. 2017), and mechanistic studies in animal models of 378 helminth infections are necessary to dissect the causality of these relationships (ㄷ. Cortés et al. 2018). Importantly, minimising variations between studies is crucial to warrant meaningful comparisons between datasets.

Whilst reducing the variability amongst samples collected from naturally helminth-infected humans may be difficult to achieve, the enormous impact that differences in technical and experimental approaches (from sample collection to bioinformatics and biostatistical analyseis) exert on the overall variation detected across studies can be reduced (Figs. 1 and 2; Lindgreen $e t$ al. 2017; Costea et al. 2017; Golob et al. 2017). In particular, a range of bioinformatics pipelines are available for the analysis of high-throughput amplicon and metagenomics sequence datasets that include, e.g., different sequence-processing tools and reference databases for sequence annotation that could yield slightly different results (Lindgreen et al. 2017; Golob et al. 2017). For instance, the use of validated open microbiome analysis packages such Multiplexed Analysis of Projections by Sequencing (MAPseq) (Matias Rodrigues et al. 2017) or QIIME2 (https://qiime2.org/) taxonomy classification of $16 \mathrm{~S}$ amplicon datasets, for instance, current trends indicate that optimised approaches should rely on open microbiome analysis packages such

QIIME2 (https://qiime2.0rg/), which have proven fast, accurate and specific in predicting 
comprehensive, as well as-regularly updated reference databases. Amongst these, , e.g.SILVA

(https://www.arb-silva.de/) (Quast et al. 2013), that enables a-sensitive annotations of bacterial rRNA sequence data (Almeida et al. 2018). Thus, the use of such standardized analysis workflows and eentinuously updated-reference databases for sequence annotation might prove extremely useful to increase consistency across studies and enable researchers to identify common and/or unique features between the gut microbiota of different host-parasite systems which, in turn, might assist to better understand the mechanisms that regulate helminth-microbiota relationships.

The consequences that elucidating such mechanisms may exert on future strategies of parasite control are two-fold. First, disentangling the potential contribution of the gut flora to the pathogenesis of the infection is necessary in order to discover and develop new strategies to contrast helminth-associated pathology. Second, understanding the microbiota-dependent mechanisms by which parasitic helminths are able to modulate host immune responses and suppress inflammation may assist the discovery of novel immune-regulatory therapeutics against chronic inflammatory disorders of the GI tract that may act in synergy with helminth-based therapy (see Peachey et al. 2017 and Rapin and Harris, 2018 for reviews). However, in order for this new knowledge to be fully exploited in translational research, further studies that thoroughly consider inclusion/exclusion criteria for the selection of participants, include appropriate controls, and follow standardised experimental and data analysis protocols $;$ are necessary, thus allowingand will allow to disentangle the potential influence of parasite-, drug- and/or population-dependent variables in each setting (Fig. 2), are necessary. 


\section{ACKNOWLEDGEMENTS}

421 The authors would like to thanks Professor R. Stephen Phillips for helpful suggestions on the draft 422 manuscript.

\section{FINANCIAL SUPPORT}

424 AC is supported by a postdoctoral fellowship from Fundación Alfonso Martín Escudero (Madrid, 425 Spain). LEP is supported by funding from the Horserace Betting Levy Board (HBLB) and TPJ 426 by scholarships by the Biotechnology and Biological Sciences Research Council (BBSRC) of the 427 United Kingdom. Research in the CC laboratory is supported by grants by the Royal Society and 428 the Isaac Newton Trust. 


\section{REFERENCES}

Almeidla A, Mitchell AL, Tarkowska A and Finn RD (2018) Benchmarking taxonomic assignments based on 16S rRNA gene profiling of the microbiota from commonly sampled environments. GigaScience 7, 1-10.

Barbour AD and Kafetzaki M (1991) Modeling the overdispersion of parasite loads. Mathematical Biosciences 107, 249-253.

Broadhurst MJ, Ardeshir A, Kanwar B, Mirpuri J, Gundra UM, Leung JM, Wiens KE, Vujkovic-Cvijin I, Kim CC, Yarovinsky F, Lerche NW, McCune JM and Loke P (2012) Therapeutic helminth infection of macaques with idiopathic chronic diarrhea alters the inflammatory signature and mucosal microbiota of the colon. PLoS Pathogens $\mathbf{8}$, e1003000.

Brosschot TP and Reynolds LA (2018) The impact of a helminth-modified microbiome on host immunity. Mucosal Immunology 11, 1039-1046.

Campbell SJ, Biritwum NK, Woods G, Velleman Y, Fleming F and Stothard JR (2018) Tailoring water, sanitation, and hygiene (WASH) targets for soil-transmitted helminthiasis and schistosomiasis control. Trends in Parasitology 34, 53-63.

Cantacessi C, Giacomin P, Croese J, Zakrzewski M, Sotillo J, McCann L, Nolan MJ, Mitreva M, Krause L and Loukas A (2014) Impact of experimental hookworm infection on the human gut microbiota. The Journal of Infectious Diseases 210, 1431-1434.

Cattadori IM, Sebastian A, Hao H, Katani R, Albert I, Eilertson KE, Kapur V, Pathak A and Mitchell S (2016) Impact of helminth infections and nutritional constraints on the small intestine microbiota. PLoS One 11, e0159770.

Chabé M, Lokmer A and Segurel L (2017) Gut protozoa: friends or foes of the human gut microbiota? Trends in Parasitology 33, 925-934.

Churcher TS, Ferguson NM and Basáñez MG (2005) Density dependence and overdispersion in the transmission of helminth parasites. Parasitology 131(Pt 1), 121-132. 
Cliffe LJ and Grencis RK (2004) The Trichuris muris system: a paradigm of resistance and susceptibility to intestinal nematode infection. Advances in Parasitology 57, 255-307. human infections with the whipworm, Trichuris trichiura, are not associated with alterations in the faecal microbiota. PLoS One 8, e76573.

Cortés A, Toledo R and Cantacessi C (2018) Classic models for new perspectives: delving into helminth-microbiota-immune system interactions. Trends in Parasitology 34, 640-654. sample processing in metagenomic studies. Nature Biotechnology 35, 1069-1076. Andrighetto I and Segato S (2016) Metagenomic analysis of rumen microbial population in dairy heifers fed a high grain diet supplemented with dicarboxylic acids or polyphenols. $B M C$ Veterinary Research 12, 29-016-0653-4.

Duarte AM, Jenkins TP, Latrofa MS, Giannelli A, Papadopoulos E, de Carvalho LM, Nolan MJ, Otranto D and Cantacessi C (2016) Helminth infections and gut microbiota - a feline 478 perspective. Parasites \& Vectors 9, 625-016-1908-4. 
481

482

483

484

485

486

487

488

489

490

491

492

493

494

495

496

497

498

499

500

501

502

503

504

505

gluten challenges are associated with increased microbial richness in celiac subjects. Scientific Reports 5, 13797.

Giacomin P, Zakrzewski M, Jenkins TP, Su X, Al-Hallaf R, Croese J, de Vries S, Grant A, Mitreva M, Loukas A, Krause L and Cantacessi C (2016) Changes in duodenal tissueassociated microbiota following hookworm infection and consecutive gluten challenges in humans with coeliac disease. Scientific Reports 6, 36797.

Glendinning L, Nausch N, Free A, Taylor DW and Mutapi F (2014) The microbiota and helminths: sharing the same niche in the human host. Parasitology 141, 1255-1271.

Golob JL, Margolis E, Hoffman NG and Fredricks DN (2017) Evaluating the accuracy of amplicon-based microbiome computational pipelines on simulated human gut microbial communities. BMC Bioinformatics 18, 283-017-1690-0.

Greetham HL, Gibson GR, Giffard C, Hippe H, Merkhoffer B, Steiner U, Falsen E and Collins MD (2004) Allobaculum stercoricanis gen. nov., sp. nov., isolated from canine feces. Anaerobe 10, 301-307.

Holm JB, Sorobetea D, Kiilerich P, Ramayo-Caldas Y, Estelle J, Ma T, Madsen L, Kristiansen K and Svensson-Frej M(2015) Chronic Trichuris muris infection decreases diversity of the intestinal microbiota and concomitantly increases the abundance of Lactobacilli. PLoS One 10, e0125495.

Hotez PJ, Alvarado M, Basanez MG, Bolliger I, Bourne R, Boussinesq M, Brooker SJ, Brown AS, Buckle G, Budke CM, Carabin H, Coffeng LE, Fevre EM, Furst T, Halasa YA, Jasrasaria R, Johns NE, Keiser J, King CH, Lozano R, Murdoch ME, O'Hanlon S, Pion SD, Pullan RL, Ramaiah KD, Roberts T, Shepard DS, Smith JL, Stolk WA, Undurraga EA, Utzinger J, Wang M, Murray CJ and Naghavi M (2014) The global burden of disease study 2010: interpretation and implications for the neglected tropical diseases. PLoS Neglected Tropical Diseases 8, e2865. 
IS (2015) Chronic Trichuris muris infection in C57BL/6 mice causes significant changes in host microbiota and metabolome: effects reversed by pathogen clearance. PLoS One 10, e0125945. microbiome and metabolome of Strongyloides stercoralis infected volunteers from a nonendemic area. Scientific Reports 8, 15651.

Jenkins TP, Peachey LE, Ajami NJ, MacDonald AS, Hsieh MH, Brindley PJ, Cantacessi C and Rinaldi G (2018a) Schistosoma mansoni infection is associated with quantitative and qualitative modifications of the mammalian intestinal microbiota. Scientific Reports 8, 12072. and Cantacessi C (2017) Infections by human gastrointestinal helminths are associated with changes in faecal microbiota diversity and composition. PLoS One 12, e0184719.

Kay GL, Millard A, Sergeant MJ, Midzi N, Gwisai R, Mduluza T, Ivens A, Nausch N, Mutapi F and Pallen M(2015) Differences in the faecal microbiome in Schistosoma haematobium infected children vs. uninfected children. PLoS Neglected Tropical Diseases 9, e0003861.

Kelly BJ, Gross R, Bittinger K, Sherrill-Mix S, Lewis JD, Collman RG, Bushman FD and Li H (2015) Power and sample-size estimation for microbiome studies using pairwise distances and PERMANOVA. Bioinformatics 31, 2461-2468.

Kim JY, Kim EM, Yi MH, Lee J, Lee S, Hwang Y, Yong D, Sohn WM and Yong TS (2018) Intestinal fluke Metagonimus yokogawai infection increases probiotic Lactobacillus in mouse cecum. Experimental Parasitology 193, 45-50. 
532

533

534

535

536

537

538

539

540

541

542

543

544

545

546

547

548

549

550

551

552

553

554

555

556

Leung JM, Graham AL and Knowles SCL (2018) Parasite-microbiota interactions with the vertebrate gut: synthesis through an ecological lens. Frontiers in Microbiology 9, 843.

Ley RE, Hamady M, Lozupone C, Turnbaugh PJ, Ramey RR, Bircher JS, Schlegel ML, Tucker TA, Schrenzel MD, Knight R and Gordon JI (2008) Evolution of mammals and their gut microbes. Science 320, 1647-1651.

Li RW, Li W, Sun J, Yu P, Baldwin RL and Urban JF (2016) The effect of helminth infection on the microbial composition and structure of the caprine abomasal microbiome. Scientific Reports 6, 20606.

Lindgreen S, Adair KL and Gardner PP (2016) An evaluation of the accuracy and speed of metagenome analysis tools. Scientific Reports 6, 19233.

Lozupone CA, Stombaugh JI, Gordon JI, Jansson JK and Knight R (2012) Diversity, stability and resilience of the human gut microbiota. Nature 489, 220-230.

Martin I, Djuardi Y, Sartono E, Rosa BA, Supali T, Mitreva M, Houwing-Duistermaat JJ and Yazdanbakhsh M (2018) Dynamic changes in human-gut microbiome in relation to a placebo-controlled anthelminthic trial in Indonesia. PLoS Neglected Tropical Diseases 12, e0006620.

Mutapi F (2015) The gut microbiome in the helminth infected host. Trends in Parasitology 31, 405-406.

$\underline{\text { Matias Rodrigues JF, Schmidlt TSB, Tackmann J and von Mering C (2017) MAPseq: highly }}$ efficient k-mer search with confidence estimates, for rRNA sequence analysis. Bioinformatics $\mathbf{3 3}$, $\underline{3808-3810 .}$

O'Connell EM and Nutman TB(2016) Molecular diagnostics for soil-transmitted helminths. The American Journal of Tropical Medicine and Hygiene 95, 508-513.

Peachey LE, Jenkins TP and Cantacessi C (2017) This gut ain't big enough for both of us. Or is it? Helminth-microbiota interactions in veterinary species. Trends in Parasitology 33, 619-632. 

Cantacessi C (2018) The relationships between faecal egg counts and gut microbial composition in UK thoroughbreds infected by cyathostomins. International Journal for Parasitology 48, 403412.

Pearce EJ and MacDonald AS (2002). The immunobiology of schistosomiasis. Nature Reviews Immunology 2, 499-511.

Pérez-Muñoz ME, Bergstrom K, Peng V, Schmaltz R, Jiménez-Cardona R, Marsteller N, McGee S, Clavel T, Ley R, Fu J, Xia L and Peterson DA (2014) Discordance between changes in the gut microbiota and pathogenicity in a mouse model of spontaneous colitis. Gut Microbes $\mathbf{5}$, 286-295.

Quast C, Pruesse E, Yilmaz P, Gerken J, Schweer T, Yarza P, Peplies J and Glöckner FO (2013) The SILVA ribosomal RNA gene database project: improved data processing and webbased tools. Nucleic Acids Research 41, 590-596.

Ramanan D, Bowcutt R, Lee SC, Tang MS, Kurtz ZD, Ding Y, Honda K, Gause WC, Blaser

MJ, Bonneau RA, Lim YA, Loke P and Cadwell K (2016) Helminth infection promotes colonization resistance via type 2 immunity. Science 352, 608-612.

Rapin A and Harris NL (2018) Helminth-bacterial interactions: cause and consequence. Trends in Immunology 39, 724-733.

$\underline{\text { Rausch S, Held J, Fischer A, Heimesaat MM, Kühl AA, Bereswill S and Hartmann S (2013) }}$ $\underline{\text { Small intestinal nematode infection of mice is associated with increased enterobacterial loads }}$ alongside the intestinal tract. PLoS One 8, e74026.

Reynolds LA, Smith KA, Filbey KJ, Harcus Y, Hewitson JP, Redpath SA, Valdez Y, Yebra MJ, Finlay BB and Maizels RM (2014) Commensal-pathogen interactions in the intestinal tract: Lactobacilli promote infection with, and are promoted by, helminth parasites. Gut Microbes $\mathbf{5}$, $522-532$.

Rosa BA, Supali T, Gankpala L, Djuardi Y, Sartono E, Zhou Y, Fischer K, Martin J, Tyagi R, Bolay FK, Fischer PU, Yazdanbakhsh M and Mitreva M (2018) Differential human gut microbiome assemblages during soil-transmitted helminth infections in Indonesia and Liberia. Microbiome 6, 33-018-0416-5. 
14

Schneeberger PHH, Coulibaly JT, Panic G, Daubenberger C, Gueuning M, Frey JE and Keiser J (2018a) Investigations on the interplays between Schistosoma mansoni, praziquantel and the gut microbiome. Parasites \& Vectors 11, 168-018-2739-2.

Sekirov I, Russell SL, Antunes LC and Finlay BB (2010) Gut microbiota in health and disease. Physiological Reviews 90, 859-904.

Stensvold CR and van der Giezen M (2018) Associations between gut microbiota and common luminal intestinal parasites. Trends in Parasitology 34, 369-377.

Su C, Su L, Li Y, Long SR, Chang J, Zhang W, Walker WA, Xavier RJ, Cherayil BJ and Shi HN (2018) Helminth-induced alterations of the gut microbiota exacerbate bacterial colitis. Mucosal Immunology 11, 144-157.

Wang J, Tang H, Zhang C, Zhao Y, Derrien M, Rocher E, van-Hylckama Vlieg JE, Strissel K, Zhao L, Obin M and Shen J (2015) Modulation of gut microbiota during probiotic-mediated attenuation of metabolic syndrome in high fat diet-fed mice. The ISME Journal 9, 1-15.

Wang WL, Xu SY, Ren ZG, Tao L, Jiang JW and Zheng SS (2015) Application of metagenomics in the human gut microbiome. World Journal of Gastroenterology 21, 803-814.

World Health Organisation (2006) Preventive chemotherapy in human helminthiasis: coordinated use of anthelminthic drugs in control interventions: a manual for health professionals and programme managers. Geneva: WHO; 2006.

Wu S, Li RW, Li W, Beshah E, Dawson HD and Urban JF Jr. (2012) Worm burden-dependent disruption of the porcine colon microbiota by Trichuris suis infection. PLoS One 7, e35470.

\section{Yatsunenko T, Rey FE, Manary MJ, Trehan I, Domínguez-Bello MG, Contreras M, Magris} M, Hidalgo G, Baldassano RN, Anokhin AP, Heath AC, Warner B, Reeder J, Kuczynski J, Caporaso JG, Lozupone CA, Lauber C, Clemente JC, Knights D, Knight R and Gordon JI (2012) Human gut microbiome viewed across age and geography. Nature 486, 222-227. 


\section{FIGURE LEGENDS}

617 Fig. 1 Sources of variation and confounding factors potentially impacting the outcome of studies 618 of human-helminth-gut microbiota interactions in helminth-endemic regions.

619 Fig. 2 Proposed approaches aimed at reducing the methodological sources of variation 620 surrounding investigations of human-helminth-gut microbiota interactions. 